\title{
The effect of Zingiber officinale R. rhizomes (ginger) on plasma pro-inflammatory cytokine levels in well-trained male endurance runners
}

\author{
FARZAD ZEHSAZ, NEGIN FARHANGI, LAMIA MIRHEIDARI
}

Department of Physical Education \& Sport Sciences, College of Humanities and Educational Sciences, Tabriz Branch, Islamic Azad University, Tabriz, Iran

\begin{abstract}
The aim of this study was to investigate the effect of ginger on the concentrations of plasma interleukin-1 $\beta(I L-1 \beta)$, interleukin-6 (IL-6) and tumor necrosis factor- $\alpha(T N F-\alpha)$ in welltrained male endurance runners. To this end, twenty-eight high-level endurance runners were randomly assigned to two groups (control and experimental). They performed the same weekly training volume for 12 weeks. The Bruce treadmill test was used three days before the start of the 12-week training period and after weeks 6 and 12 to evaluate the physiological effects of training and ginger administration. After completing the first 6-week period of exercise training protocol, two groups received $500 \mathrm{mg}$ capsules of ginger powder and placebo, respectively, three times a day for the second 6-week period. Blood samples were collected before (resting blood sample) and after the Bruce treadmill test. $I L-1 \beta, I L-6$ and TNF- $\alpha$ were assayed using standard commercial ELISA kits. Two-way repeated measures analysis of variance (ANOVA) was used to determine significant differences between control and experimental plasma IL-6, $I L-1 \beta$ and TNF- $\alpha$ concentration means of pre- and post-test periods. The $I L-1 \beta, I L-6$ and TNF- $\alpha$ concentrations at the end of week 12/weeks 6 and 12 between two training groups were significantly different $(p=0.01, p=0.01$, and $p=0.01)$. In the experimental group alone, there were also significant differences in the mean IL-1 $\beta, I L-6$ and TNF- $\alpha$ concentrations at the end of weeks 6 and $12(p=0.01, p=0.01$, and $p=0.01)$. Our findings indicated that post-exercise plasma elevations of several pro-inflammatory cytokines can be attenuated by a six-week ginger administration period.
\end{abstract}

Key words: endurance training, immune, ginger, interleukin-1 $\beta$, interleukin-6, tumor necrosis factor- $\alpha$

(Centr Eur J Immunol 2014; 39 (2): 174-180)

\section{Introduction}

Prolonged aerobic exercise induces a large systemic inflammatory response [1-2], which may lead to an infection due to a suppression of the immune system. For example, the prevalence of upper respiratory infections in athletes is increased following a prolonged aerobic event [3, 4]. Prolonged exercise causes an inflammatory response that is expressed by an increase in circulating anti-inflammatory and pro-inflammatory cytokines. Thus, moderate activity may enhance the immune's functional capacity, while prolonged and intense exercise may impair the immune function, which finally results in a decline in exercise performance and the ability to undergo heavy training [5].
Cytokines are hormone-like proteins that mediate inflammatory responses by autocrine, paracrine and endocrine effects and can be considered markers of inflammation. Strenuous exercise, energy crisis, stress hormones and oxidative stress are examples of physiological stimuli that modulate cytokine production. Cytokines, including tumour necrosis factor $\alpha$ (TNF- $\alpha)$, interleukin- $1 \beta$ (IL-1 $\beta$ ), interleukin-6 (IL-6), interferon $\gamma($ IFN- $\gamma$ ) and interleukin-10 (IL-10), are largely responsible for dictating profound physiological changes both locally and systemically [6].

Most researchers believe that the prolonged exercise induces systemic pro-inflammatory responses and that pro-inflammatory cytokines such as TNF- $\alpha$ increase and

Correspondence: Farzad Zehsaz, Department of Physical Education \& Sport Sciences, College of Humanities and Educational Sciences, Tabriz Branch, Islamic Azad University, T 5157944533 Tabriz, Iran, e-mail: f-zehsaz@iaut.ac.ir 
remain elevated in the plasma for at least about 2 hours. They have further demonstrated that increased circulating levels of TNF- $\alpha$ are positively related to the rate of infection [7]. Ostrowsky et al. (1998) also showed 2.5 fold increases in the plasma TNF- $\alpha$ levels after 2.5 hours of the endurance training at moderate to high intensity [8]. They suggested that muscle injury consequent to prolonged endurance exercise might play an important role in this response. It is difficult to determine the exact magnitude of physical training stress needed to induce a significant increase in circulation pro-inflammatory cytokines since athlete responses are not homogenous and are affected by many physiological, psychological and training-related factors. Studies have also found up to 100-fold IL-6 increase after endurance events [9]. This increase in circulating IL-6 concentration in athletes is similar to that seen in patients with infections [10]. Although some physiological roles IL-1 $\beta$, IL- 6 and TNF- $\alpha$ overlap, they also exhibit distinctly different functions. Dysregulation of the inflammatory response resulting in excessive production of pro-inflammatory cytokines or their production in the wrong biological context may lead to chronic inflammation, which can be life threatening in extreme cases [11].

Since ancient times, in various cultures worldwide, inflammatory disorders and related diseases have been treated with plants or plant-derived formulations [12-13]. Ginger has long been used in traditional medicine as a cure for some diseases including inflammatory diseases. Ginger contains active phenolic compounds such as gingerol, paradol and shogoal that have antioxidant, anti-cancer, anti-inflammatory, anti-angiogenesis and anti-atherosclerotic properties [14].

The anti-inflammatory properties of ginger have been known and valued for centuries. The original discovery of ginger's inhibitory effects on prostaglandin biosynthesis in the early 1970 s has been repeatedly confirmed. This discovery identified ginger as a herbal medicinal product that shares pharmacological properties with non-steroidal anti-inflammatory drugs [15]. The discovery preceded the observation that dual inhibitors of cyclooxygenase and 5-lipoxygenase may have a better therapeutic profile and have fewer side effects than non-steroidal anti-inflammatory drugs [15].

The primary purpose of this study was to investigate the effect of ginger administration on the concentration of post-exercise plasma levels of IL-1 $\beta$, IL- 6 and TNF- $\alpha$ in athletes. We were interested in determining if ginger administration could improve indicators of athletes' acute immune function without compromising the taper induced benefits to their performance capacity. We were particularly interested in the post-exercise values as these are reflective of the acute stress of the exercise on cytokine production. While monitoring of chronic non-exercise induced in plasma cytokine levels would have further informed this study, we were not able to perform these measures in the study design.

\section{Material and methods}

\section{Subjects}

Twenty-eight well-trained male endurance runners participating in the national league volunteered to participate in this study as subjects. They were informed of the purposes and methods of the study before giving written consent to participate. Subjects had been free from infection for 6 weeks before the study, were exempt from symptoms of respiratory illnesses, and were not taking any medication (medical drugs, nutritional supplements, vitamins, herbs). The experimental protocol was approved by the Human Research Ethics Committee of the IA University (No. 2/25-2014). Before the beginning of 12 weeks' training protocol, the subjects were randomly assigned to two groups (control and experimental groups). Table 1 gives the physical and training characteristics of the subjects at the start of the study.

\section{Exercise program}

To induce realistic training programs, twenty-eight well-trained endurance runners were fully trained as if preparing for a competition season. During 12 weeks, the control and experimental groups performed the same training regimen together. The training status of every subject over the preceding 12 weeks and their training histories were obtained using a questionnaire, training records, and personal interview. Runners were considered as well trained if they had trained for at least 3 years, 2 hours a day, 4 to 5 times a week. Only subjects who met these criteria were included in the study.

\section{Running performance measurement and $\mathrm{VO}_{2 \max }$ assessment}

Three days before the start of the 12-week training period, each subject performed the Bruce treadmill test. The subjects reported to the laboratory at $08.00 \mathrm{~h}$, after an overnight fast. Subjects were allowed to drink water ad libitum, but consumption of carbohydrates was not allowed until after running. Subjects had been instructed to arrive well

Table 1. Demographic, anthropometric and sports activity data for the control and experimental group athletes at the start of the study. Data are means $( \pm$ SD)

\begin{tabular}{lcc}
\hline Variable & Experimental & Control \\
\hline Height $(\mathrm{cm})$ & $1.72 .93(5.48)$ & $1.74 .57(3.97)$ \\
\hline Weight $(\mathrm{kg})$ & $65.36(2.87)$ & $64.93(4.34)$ \\
\hline Age $($ years $)$ & $23.21(2.77)$ & $22.03(2.34)$ \\
\hline $\mathrm{VO}_{2 \mathrm{max}}\left(\mathrm{ml} \cdot \mathrm{kg}^{-1} \cdot \mathrm{min}^{-1}\right)$ & $67.86(0.75)$ & $66.79(1.98)$ \\
\hline Sport experience $($ years $)$ & $5.14(1.70)$ & $4.57(1.78)$ \\
\hline
\end{tabular}


rested and to refrain from exhaustive training in the days before the experiment. On entering the laboratory, both control and experimental groups lay on a bench and rested for 15 minutes. The lowest heart rate during that period was recorded. Then, they ran on a treadmill (Ergo XELG 90; Woodway, Weil, Germany) to perform the Bruce treadmill test as instructed in Bruce's (1972) [16] report. The Bruce Protocol is a maximal exercise test whereas the athlete works to complete exhaustion as the treadmill speed and incline is increased every three minutes. The length of time on the treadmill is the test score and can be used to estimate the $\mathrm{VO}_{2 \max }$ value [17]. Expired gases were collected and analyzed by open circuit spirometry (Sensor Medics, Yorba Linda, CA) by using an automated metabolic analysis system. The data were averaged in $20 \mathrm{sec}$ intervals. The gas analyzer was calibrated with primary standard gas $(16.0 \%$ $\mathrm{O}_{2}, 4.0 \% \mathrm{CO}_{2}$, balance $\mathrm{N}_{2}$ ) before each test [18].

The Bruce treadmill test was used as the standard test (i.e., index of performance) three days before the start of the 12-week training period and after weeks 6 and 12 (before, during and after training period) to evaluate the physiological effects of training and ginger administration. Each runner was asked to complete the performance test as fast as possible with no information provided on how well he was performing until the end of the test.

\section{Intervention}

Zingiber officinale $\mathrm{R}$. rhizomes were collected in April 2013 from the Institute of Medicinal Plants field. A voucher specimen of the plant (number 1483) has been deposited in the Central Herbarium of the Institute. The rhizomes were dried in a dark condition at room temperature and ground to a powder that was encapsulated [19].

\section{Preparation of Ginger and placebo capsules}

The placebo capsules contained toast powder. The capsules were similar in shape, taste and colour but one set contained $500 \mathrm{mg}$ of ginger powder per capsule and the others were placebo capsules. The ginger capsules did not have a distinguishable smell. The capsules were prepared at the Institute of Medicinal Plants and put into coded packages. Capsules and their packages were identical in appearance. The authors of this research used double-blind procedures, therefore after completing the first 6-week period of exercise training protocol, the ginger (experimental) and placebo (control) groups received $500 \mathrm{mg}$ capsules of ginger powder and placebo, respectively, three times a day for the second 6-week period.

\section{Blood sampling}

Blood samples were collected from 28 participants three days before the start of the 12-week training period and after weeks 6 and 12, before (resting blood sample) and immediately after the Bruce treadmill test. Venous blood samples were drawn from a forearm vein into sodium heparin tubes chilled on ice. Blood was centrifuged at $700 \mathrm{rpm}$ at $23^{\circ} \mathrm{C}$ for $10 \mathrm{~min}$. Plasma was separated and stored at $-70^{\circ} \mathrm{C}$.

\section{Plasma cytokine measurements}

TNF- $\alpha$, IL- $1 \beta$ and IL-6 levels were analysed in duplicate with all values expressed as a mean of the two determinations, using validated ELISA kits (Quantikine; R \& D Systems, Minneapolis, MN). The minimum detectable dose (MDD) of TNF- $\alpha$, IL- $1 \beta$ and IL- 6 ranged from $0.038 \mathrm{pg} / \mathrm{ml}$ to $0.191 \mathrm{pg} / \mathrm{ml}$, less than $1.0 \mathrm{pg} / \mathrm{ml}$ and from 0.016 to $0.110 \mathrm{pg} / \mathrm{ml}$, respectively. A standard curve was made using standards provided in the kits, and the cytokine concentrations were appointed from the standard curves by use of linear regression analysis. The assays were a two-step "sandwich" enzyme immunoassay in which samples and standards were incubated in a 96-well microtiter plate coated with polyclonal antibodies for the test cytokine as the capture antibody. After the proper incubation time, the wells were washed and a second detection antibody conjugated to either alkaline phosphatase (IL-6 high sensitivity) or horseradish peroxidase (TNF- $\alpha$ and IL-1 $\beta$ ) was added. The plates were incubated and washed, and the amount of bound enzyme-labelled detection antibody was measured by adding a chromogenic substrate. The plates were then read at the proper wave length (450 minus $570 \mathrm{~nm}$ for TNF- $\alpha$ and IL-1 $\beta ; 490$ minus $650 \mathrm{~nm}$ for IL-6 high sensitivity). The minimum detectable doses of IL-1 $\beta$, IL- 6 and TNF- $\alpha$ are typically less than 1.0, 0.016 -0.110 and $0.038-0.191 \mathrm{pg} / \mathrm{ml}$, respectively.

\section{Statistical analysis}

Means and standard deviations were used to describe quantitative variables. Two-way repeated measures analysis of variance (ANOVA) was used to determine significant differences between control and experimental plasma IL-6, IL- $1 \beta$ and TNF- $\alpha$ concentration means of pre- and post-test periods. Also, significant differences between pre- and post-test $(\Delta)$ IL- 6 , IL- $1 \beta$ and TNF- $\alpha$ concentrations were determined using two-way repeated measures analysis of variance (ANOVA). Data in text and figures are given as the mean \pm SEM. $P \leq 0.05$ is considered statistically significant. All data were analyzed by using SPSS for Windows software version 20.0 (SPSS Inc, Chicago, IL).

\section{Results}

There were no physical, training experience or $\mathrm{VO}_{2 \max }$ differences between the control or taper/experimental group subjects (see Table 1). All 28 subjects completed the 12-week training period and the data from all subjects were included in the analyses. Figure 1 indicates the comparison 
of the means (SD) of plasma IL-1 $\beta$, IL-6 and TNF- $\alpha$ concentrations in the control and experimental groups.

Within control and experimental groups, there were no significant differences in the rest IL-1 $\beta$ concentrations at three collecting periods $(F=0.54, p=0.59$, and $F=0.69$, $p=0.51$, respectively). Additionally, there were no significant differences in the IL- $1 \beta$ concentrations between two training groups on the day before beginning the training protocol and after 6 weeks of it $(p=0.40$, and $p=0.30$, respectively). However, the IL-1 $\beta$ concentrations at the end of week 12/weeks 6 and 12 between two training groups were significantly different $(p=0.01)$. In the experimental group alone, there were also significant differences in the mean IL-1 $\beta$ concentrations at the end of weeks 6 and 12 $(p<0.01)$ and means at the end of week 12 were $18.95 \%$ lesser than those at the end of week 6. In the control group, however, there were significant differences in the mean IL- $1 \beta$ concentrations at the end of weeks 6 and $12(p<0.01)$, but the means at the end of week 12 were $32.04 \%$ greater than those at the end of week 6.

Within control and experimental groups, there were no significant differences in the rest IL-6 concentrations at three collecting periods $(p=0.17$, and $p=0.19$, respectively). In addition, there were no significant differences in the IL- 6 concentrations between two training groups on the day before beginning the training protocol and after 6 weeks of it ( $p=0.64$, and $p=0.051$, respectively). However, the IL- 6 concentrations between two training groups were significantly different $(p=0.01)$ at the end of week 12 . In the experimental group alone, there were also significant differences in the mean IL- 6 concentrations at the end of weeks 6 and $12(p<0.01)$ and means at the end of week 12 were $17.5 \%$ lesser than those at the end of week 6 . In the control group, however, there were significant differences in the mean IL- 6 concentrations at the end of weeks 6 and $12(p<0.01)$, but $22.04 \%$ increases were observed when comparing levels of IL- 6 at the end of week 6 with those at the end of week 12 in the experimental group.

Within control and experimental groups, there were no significant differences in the rest IL- $1 \beta$ concentrations at three collecting periods $(p=0.71$, and $p=0.18$, respectively). Additionally, plasma TNF- $\alpha$ levels between the two training groups showed no significant differences on the day before beginning the training protocol and after 6 weeks of it ( $p=0.14$, and $p=0.09$, respectively). However, the TNF- $\alpha$ concentrations between two training groups were significantly different $(p=0.01)$ at the end of week 12. In the experimental group alone, there were also significant differences in the mean TNF- $\alpha$ concentrations at the end of weeks 6 and $12(p<0.01)$ and means at the end of week 12 were $11.14 \%$ lesser than those at the end of week 6 . In the control group, however, there were significant differences in the mean TNF- $\alpha$ concentrations at the end of weeks 6 and $12(p<0.01)$, but $10.14 \%$ increases were observed when comparing levels of TNF- $\alpha$ at the end of week 6 with those at the end of week 12 in the experimental group. See Fig. 1 for all of the above cytokine data.

\section{Discussion}

This study demonstrates that post-exercise plasma elevations of several pro-inflammatory cytokines can be attenuated by a six-week ginger administration period, which will elicit improvements in the immune function in well-trained runners relative to those who continue heavy training. This study examined the effect of 6 weeks of ginger administration in which training volume was increased.

Our findings indicated that continuous training sessions performed in this study may induce increases in circulating pro-inflammatory cytokines immediately post-exercise suggesting that intense training performed by runners in this study may have temporarily compromised their immune system [20]. Previous studies have reported that 10 to 30 percent of professional athletes exhibited negative physiological signs indicative of increased training volume during final preparation for championships events [21]. These symptoms have included the changes in the hypothalamus-pituitary-adrenal axis [22].

In our results, there were significant reductions in plasma IL-1 $\beta$, IL- 6 and TNF- $\alpha$ concentrations of the experimental group (ginger administration) relative to the control groups following exercise at the end of week 12 of training. While after 6 week of the progressive training program (at the end of week 6), there were no significant differences between the IL-1 $\beta$, IL-6 and TNF- $\alpha$ concentrations between the two groups. Since our measures were only performed immediately following the Bruce treadmill test, it is difficult to attribute how long these elevations may have lasted or if they also resulted in chronic resting elevations. Nevertheless, significant increases in the post-exercise IL- $1 \beta$, IL- 6 and TNF- $\alpha$ concentrations after 6 weeks of the endurance training in both groups and the continued elevation of these cytokines at 12 weeks in the control group, suggesting a greater proinflammatory profile in these athletes. Post-exercise elevation of IL-1 $\beta$, IL- 6 and TNF- $\alpha$ concentrations in the control group which continued heavy training at week 12 may be directly due to their higher training volume and that in turn may make these athletes more prone to infection [23]. Previous studies have also reported that there were significant increases in IL-1 $\beta$, IL- 6 and TNF- $\alpha$ post-exercise plasma concentrations after the prolonged endurance training $[24,25]$ that may be an indicator of acute inflammation. Elevation of these plasma cytokines is related to increased susceptibility to infection [26]. Smith has suggested that prolonged endurance exercise training may induce a long-term inflammatory state and may also indicate muscle damage [27]. Previous studies have also noted that the cytokine responses to the training are similar 
A

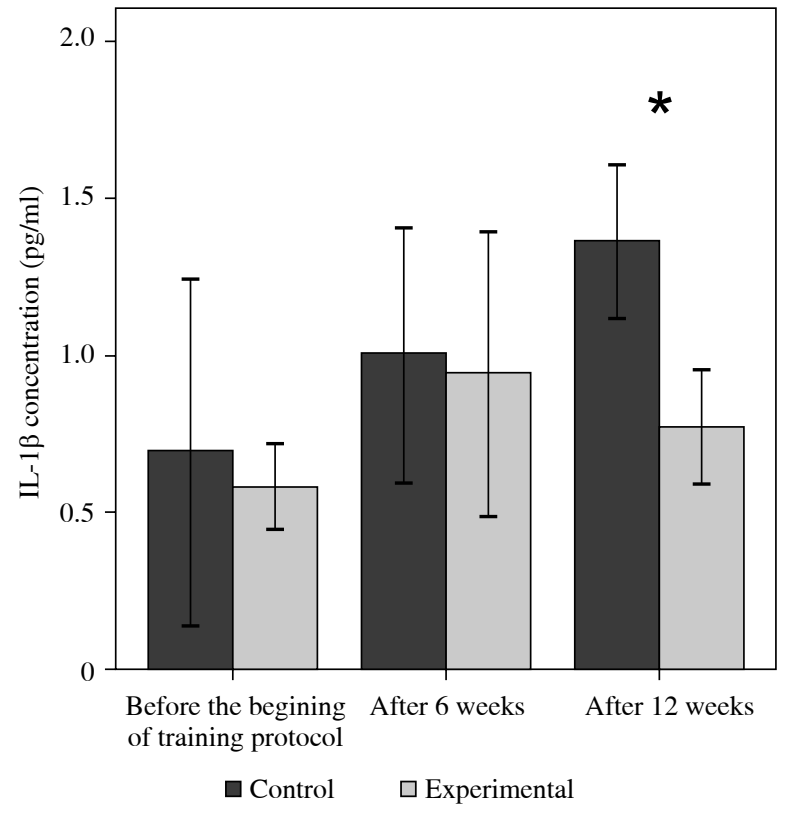

C

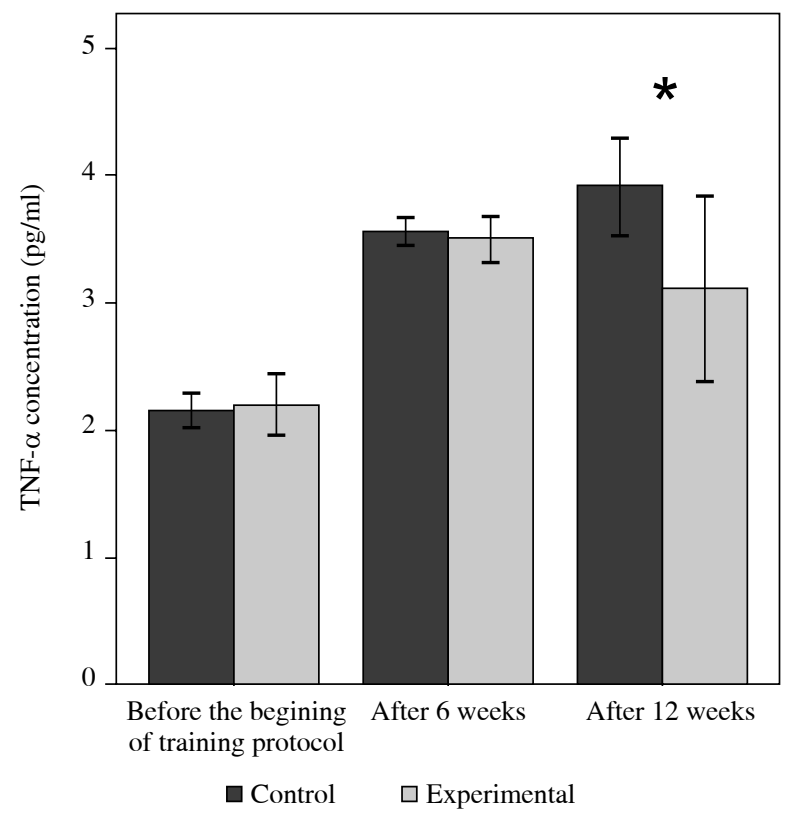

to the cytokine responses to the injuries. Clinical research has found that the cytokines also play an important role in the initiating of fatigue in disease states [28] and in the prolonged fatigue syndrome [29]. Elite athletes often suffer from the excessive and chronic fatigue and upper respiratory tract infection (URTI) and their exercise performance is consequently compromised [30].

Since inflammation is a common problem which endurance athletes are suffering these days, so our experimental study is more concentrated on an anti-inflammatory compound of ginger, particularly out of several anti-in-
B

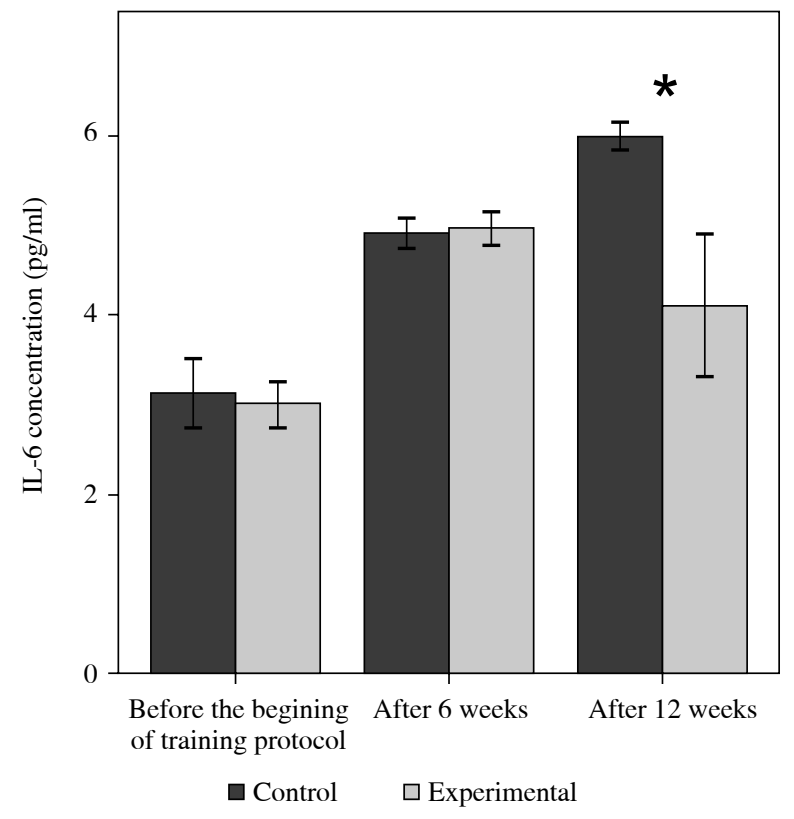

Fig. 1. The comparison of the means (SD) of the plasma IL-6, IL- $1 \beta$ and TNF- $\alpha$ concentrations in the control and experimental groups immediately after the Bruce treadmill test at various time points

flammatory ones present in Zingiber officinale (Common name: Ginger). The results of this study illustrated that the use of powdered ginger attenuated post-exercise plasma elevations of several pro-inflammatory cytokines in elite runners relative to those who performed intense training without the use of powdered ginger. In our results, there were significant reductions in plasma IL-1 $\beta$, IL-6 and TNF- $\alpha$ concentrations of the ginger using group relative to the control group following exercise at the end of week 12 of training. This finding is in agreement with Black et al.'s report which demonstrated that 11 consecutive 
days of dietary supplementation with 2 grams of raw and heat-treated ginger reduces muscle inflammation caused by eccentric exercise in athletes [31].

The anti-inflammatory properties of ginger have been known for centuries $[32,33]$. Several lines of evidence have been provided, mostly in different animal models of inflammation, and to a much lesser extent in humans or human cells, of the effectiveness of either ginger or of compounds isolated therefrom against inflammation and its mediators. In the early 1980s, it was reported for the first time that ginger has anti-inflammatory actions, as evidenced by its inhibitory effects on prostaglandins synthesis [34]. Subsequently, it has been demonstrated that ginger contains constituents (e.g. gingerdiones and shogoals) that have pharmacological properties mimicking dual-acting non-steroidal anti-inflammatory drugs (NSAIDs) in intact human leukocytes in vitro [35]. It is known that such inhibitors have fewer side effects and are more effective than conventional NSAIDs $[36,37]$. Further, it has been shown that gingerols are very active in inhibiting both prostaglandins and leukotrienes in RBL-1 cells, and that gingerols with long alkyl side-chains are more potent inhibitors of leukotrienes synthesis than of prostaglandins synthesis [38]. More recently, it has been shown that ginger (and some of its constituents) is effective against cytokines synthesized and secreted at sites of inflammation [33]. The evidence showed that Z. officinale administration suppresses inflammation through suppression of pro-inflammatory cytokines and chemokines produced by synoviocytes, chondrocytes, and leukocytes [35].

The active constituent in ginger, 6-gingerol, is an effective anti-inflammatory substance because of its inhibition of macrophage activation, more specifically by its inhibition of pro-inflammatory cytokines and antigen presentation by lipopolysaccharide-activated macrophages. It was concluded that 6-gingerol selectively inhibits production of pro-inflammatory cytokines from macrophages, but does not affect the antigen presenting cells (APC) function. Therefore, gingerol acts as an anti-inflammatory compound that may be useful to treat inflammation without interfering with the antigen presenting function of macrophages [35].

\section{Conclusions}

Our findings indicate that prolonged intense training for 6 weeks in well-trained male endurance runners can significantly elevate post-exercise plasma levels of several pro-inflammatory cytokines and that a ginger using period of 6 weeks will essentially reverse these elevations. Continuation of more intense training for 6 more weeks will not result in attenuated pro-inflammatory cytokine levels in the blood as was seen in the ginger using group. These results highlight the importance of a 6 weeks' ginger using period in proper preparation of athletes for competition since this period can modulate inflammation and reduce circulating pro-inflammatory cytokine levels in athletes, thereby possibly limiting the chances of infection and potentially reducing the effects of these cytokines in inducing fatigue-like symptoms in athletes. Therefore, it may be better if coaches apply approximately 6 weeks' ginger using periods prior to important competitions in order to properly prepare endurance athletes of optimal performance and recovery.

The authors declare no conflict of interest.

\section{References}

1. Nieman DC, Henson DA, Davis JM, et al. (2007): Quercetin's influence on exercise-induced changes in plasma cytokines and muscle and leukocyte cytokine mRNA. J Appl Physiol 103: 1728-1735.

2. Nieman DC, Konrad M, Henson DA, et al. (2012): Variance in the acute inflammatory response to prolonged cycling is linked to exercise intensity. J Interferon Cytokine Res 32: 12-17.

3. Nieman DC, Johanssen LM, Lee JW, Arabatzis K (1990): Infectious episodes in runners before and after the Los Angeles Marathon. J Sport Med Phys Fit 30: 316-328.

4. Peters EM, Goetzsche JM, Grobbelaar B, Noakes TD (1993): Vitamin C supplementation reduces the incidence of postrace symptoms of upper-respiratory-tract infection in ultramarathon runners. Am J Clin Nutr 57: 170-174.

5. Baltopoulos $P$ (2009): Exercise induced modulation of immune system functional capacity. Biology of Exercise 5: 39-50.

6. Shaikh PZ (2011): Cytokines \& their physiologic and pharmacologic functions in inflammation: A review. Int J Pharm Life Sci 2: 1247-1263.

7. Pedersen BK, Hoffman-Goetz L (2000): Exercise and the immune system: regulation, integration and adaptation. Physiol Rev 80: 1055-1081.

8. Ostrowski K, Rohde T, Zacho M, et al. (1998): Evidence that interleukin-6 is produced in human skeletal muscle during prolonged running. J Physiol 508: 949-953.

9. Ostrowski K, Rohde T, Asp S, et al. (1999): Pro- and anti-inflammatory cytokine balance in strenuous exercise in humans. J Physiol 515: 287-291.

10. Bruunsgaard H, Skinhoj P, Qvist J, Pedersen BK (1999): Elderly humans show prolonged in vivo inflammatory activity during pneumococcal infections. J Infect Dis 180: 551-554.

11. Han J, Ulevitch R (2005): Limiting inflammatory responses during activation of innate immunity - a review. Nat Immunol 6: $1198-1205$

12. Mueller M, Hobiger S, Jungbauer A (2010): Anti-inflammatory activity of extracts from fruits, herbs and spices. Food Chem 122: 987-996.

13. Rathore B, Mahdi AA, Paul BN, et al. (2007): Indian herbal medicines: Possible potent therapeutic agents for rheumatoid arthritis. J Clin Biochem Nutr 41: 12-17.

14. Habib SHM, Makpol S, Hamid NAA, et al. (2008): Ginger extract (zingiber officinale) has anti-cancer and anti-inflammatory effects on ethionine-induced hepatoma rats. Clinics 63: 807-813. 
15. Kumar S, Saxena K, Singh UN, Saxena R (2013): Anti-inflammatory action of ginger: A critical review in anemia of inflammation and its future aspects. Int J Herb Med 1: 16-20.

16. Bruce RA (1972): Multi-stage treadmill test of maximal and sub maximal exercise. Exercise Testing and Training of apparently Health Individuals: A handbook for physicians 1972.

17. Amirsasan R, Mirshafiei A, Gaeini AA, et al. (2011): Effects of Exhaustive Aerobic Exercise on Matrix Metaloproteases Activity in Athletes and Non-Athletes. World J Sport Sci 4: 185-191.

18. Rietjens GJ, Keizer HA, Kuipers H, Saris WH (2001): A reduction in training volume and intensity for 21 days does not impair performance in cyclists. Brit J Sport Med 35: 431-434.

19. Rahnama P, Montazeri A, Fallah Huseini H, et al. (2012): Effect of Zingiber officinale R. rhizomes (ginger) on pain relief in primary dysmenorrhea: a placebo randomized trial. BMC Complem Altern M 12: 1-8.

20. Ronsen O, Kjeldsen-Kragh J, Haug E (2002): Recovery time affects immunoendocrine responses to a second bout of endurance exercise. Am J Physiol Cell Ph 283: C1612-C1620.

21. Tessetore A, Meeusen R, Tiberi M, et al. (2005): Aerobic and anaerobic profiles, heart rate and match analysis in older soccer players. Ergonomics 48: 122-129.

22. Nijs J, Meeus M, Mc Gegor NR (2005): Chronic fatigue syndrome: exercise performance related to immune dysfunction. Med Sci Sport Exer 37: 1647-1654.

23. Shepley B, MacDougall JD, Cipriano N, et al. (1992): Physiological effects of tapering in highly trained athletes. J Appl Physiol 72: 706-711.

24. Brenner IKM, Natale VM, Vasiliou P (1999): Impact of three different types of exercise on components of the inflammatory response. Eur J Appl Physiol 80: 452-460.

25. Camus G, Poortmans J, Nys MI, et al. (1997): Mild endotoxemia and the inflammatory response induced by a marathon race. Clin Sci 92: 415-422.

26. Gleeson M, Nieman DC, Pedersen BK (2004): Exercise, nutrition and immune function. J Sport Sci 22: 115-125.

27. Smith LL (2000): Cytokine hypothesis of overtraining: a physiological adaptation to excessive stress? Med Sci Sport Exer 32: 317-331.

28. Nishimoto N, Sasai M, Shima Y, et al. (2000): Improvement in Castleman's disease by humanized anti-interleukin-6 recep tor antibody therapy. Blood 95: 56-61.

29. Arnold MC, Papanicolaou DA, O'Grady JA, et al. (2002): Using an interleukin- 6 challenge to evaluate neuropsychological performance in chronic fatigue syndrome. Psychol Med 32: 1075-1089.

30. Metz J (2003): Upper respiratory tract infections: who plays, who sits? Curr Sports Med Rep 2: 84-90.

31. Black CD, Herring MP, Hurley DJ, et al. (2010): Ginger (Zingiber officinale) reduces muscle pain caused by eccentric exercise. J Pain 11: 894-903.

32. Afzal M, Al-Hadidi D, Menon M, et al. (2001): Ginger: an ethnomedical, chemical and pharmacological review. Drug Metab Drug Interact 18: 159-190.

33. Grzanna R, Lindmark L, Frondoza CG (2005): Ginger - an herbal medicinal product with broad anti-inflammatory actions. J Med Food 8: 125-132.

34. Kiuchi F, Shibuya M, Sankawa U (1982): Inhibitors of prostaglandin biosynthesis from ginger. Chem Pharm Bull (Tokyo) 30: 754-757.

35. Ali BH, Blunden G, Tanira MO, Nemmar A (2008): Some phytochemical, pharmacological and toxicological properties of ginger (Zingiber officinale Roscoe): A review of recent research. Food Chem Toxicol 46: 409-420.

36. Charlier C, Michaux C (2003): Dual inhibition of cyclooxygenase-2 (COX-2) and 5-lipoxygenase (5-LOX) as a new strategy to provide safer non-steroidal anti-inflammatory drugs. Eur J Med Chem 38: 645-659.

37. Martel-Pelletier J, Lajeunesse D, Reboul P, Pelletier JP (2003): Therapeutic role of dual inhibitors of 5-LOX and COX, selective and non-selective non-steroidal anti-inflammatory drugs. Ann Rheum Dis 62: 501-509.

38. Kiuchi F, Iwakami S, Shibuya M, Hanaoka F, et al. (1992): Inhibition of prostaglandin and leukotriene biosynthesis by gingerols and diarylheptanoids. Chem Pharm Bull (Tokyo) 40: 387-391. 